
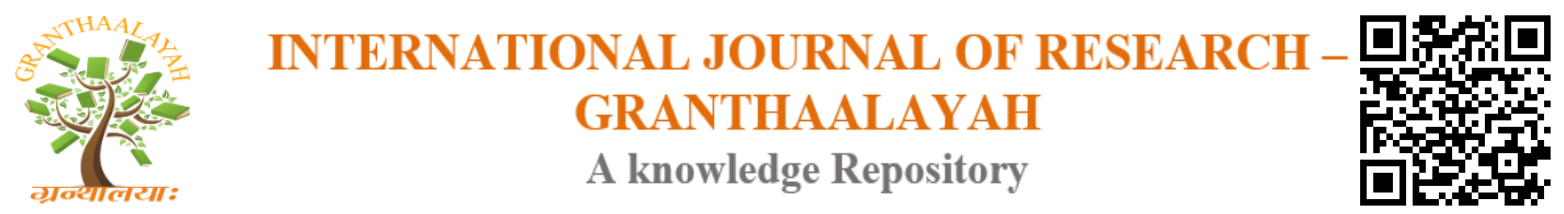

Science

\title{
COMPARATIVE ANALYSIS OF OCCLUSIVE DRESSING AND OPEN WOUND TREATMENT IN PREVENTION OF SURGICAL SITE INFECTIONS
}

\author{
Dr. Arvind Kumar Srivastava ${ }^{* 1}$, Prakshi Solanki ${ }^{2}$ \\ ${ }^{* 1}$ Association Professor, Department of Surgery, Mayo Institute of Medical Sciences, Barabanki \\ (UP), India \\ ${ }^{2}$ Intern, Department of Surgery, Mayo Institute of Medical Sciences, Barabanki (UP), India
}

\begin{abstract}
Surgical site infections (SSIs) are defined as infections of the tissues, organs, or spaces exposed by surgeons during performance of an invasive procedure.

Motivation/Background: A lot of expense both with regards to nursing time and dressing material costs is invested in prevention of SSI using occlusive surgical incision site dressing. But to what extent is this beneficial over open wound treatment? In the present study, we have compared the incidence of SSIs in surgical wounds treated with occlusive dressings versus those treated with open wound treatment to find out the same.

Method: The study was conducted on 860 patients of General Surgery wards. Patients were categorized under two groups of cases where (1) Dressing was opened after 24 hours and then only open wound treatment by cleaning with Betadine 12 hourly was done till stitch removal and (2) Dressing was opened after 48 hours and then again after every 2 days occlusive dressing was done until stitch removal.

Result: A Chi-Square Test was performed to understand if Occlusive dressings have an added advantage over Open Wound Treatment in prevention of SSIs.

Conclusion: Occlusive Dressings have no added advantage over Open Wound Treatment in the prevention of SSIs and hence Open wound treatment can be taken as an alternative for occlusive dressings.
\end{abstract}

Keywords: Surgical Site Infections; Open Wound Treatment; Occlusive Dressing.

Cite This Article: Dr. Arvind Kumar Srivastava, and Prakshi Solanki. (2019). "COMPARATIVE ANALYSIS OF OCCLUSIVE DRESSING AND OPEN WOUND TREATMENT IN PREVENTION OF SURGICAL SITE INFECTIONS." International Journal of Research - Granthaalayah, 7(9), 88-91. https://doi.org/10.29121/granthaalayah.v7.i9.2019.563.

\section{Introduction}

In this paper we compare the incidence of Surgical Site Infections in Occlusive dressing versus Open Wound treatment. From Ignaz Semmelweis, 1847, proposing chlorinated water and 
handwashing in labor rooms to Joseph Lister proposing antiseptic surgical methods in 1867 to the present day, SSIs have been regarded as a dangerous yet preventable post-surgical complication. A study conducted by A Arora et al states that while the global incidence of SSI is around $0.5 \%$ to $15 \%$, SSI in India is considerably higher ranging from $23 \%$ to $38 \%[1]$

Surgical Site Infection incidents may be influenced by surgical environment, room temperature and humidity, type of surgery, pre- and post-operative care. SSI prevention may be carried out by different surgeons by way of Occlusive dressing or Open Wound treatment. It is controversial as to which method is a better way of preventing SSIs and in order to understand the difference better, this study was conducted on 860 patients of General Surgery ward, supported by statistical methods to test the hypothesis of one procedure of SSI prevention having advantage over the other.

\section{Materials and Methods}

The study was conducted on 860 patients of General Surgery wards of Mayo Institute of Medical Sciences, Gadia, Barabanki between April 2014 to March 2019.

\section{Inclusion Criteria}

- $\quad$ Only patients undergoing elective surgical procedures were taken.

- $\quad$ The surgical wounds that are classified as Clean (in which no infection is present; only skin microflora potentially contaminate the wound, and no hollow viscus that contains microbes is entered) and Clean Contaminated (in which a hollow viscus such as the respiratory, alimentary, or genitourinary tracts with indigenous bacterial flora is opened under controlled circumstances without significant spillage of contents) were included.

\section{Exclusion Criteria}

- $\quad$ Cases of Road traffic accidents

- $\quad$ Patients undergoing Emergency surgical procedures

- $\quad$ Patients with Contaminated wounds (those with extensive introduction of bacteria into a normally sterile area of the body due to major breaks in sterile technique, gross spillage of viscus contents such as from the intestine, or incision through inflamed, albeit non-purulent tissue) or Dirty wounds (in which necrotic tissue is present, those created in the presence of overt infection as evidenced by the presence of purulent material, and those created to access a perforated viscus accompanied by a high degree of contamination).

- Patients having any of the following risk factors for development of SSIs -

1) Diabetes mellitus

2) Anemia

3) Chronic renal injury/Renal failure

4) Peripheral vascular disease

5) Steroid therapy

6) Malignancy

7) Chemotherapy or radiotherapy

8) Tuberculosis

9) AIDS

10) Burn wounds 
After taking informed consent, a detailed history taking, general physical examination and local examination, the cases fulfilling the above-mentioned criteria were included in the study.

Patients were then divided into two groups -

- $\quad$ GROUP A (430 patients) - these underwent open wound treatment.

- $\quad$ GROUP B (430 patients) - these underwent occlusive surgical dressing.

Pre-operative

- $\quad$ Part preparation was done according to the surgical procedures.

- $\quad$ Injection Ceftriaxone $1 \mathrm{gm}$ intravenous was given to all cases.

- $\quad$ Surgical sites were cleaned and painted using spirit, Savlon and Betadine in this order and then draped with sterile drapes.

Operatively - under all aseptic precautions, good haemostasis was achieved and it was insured that no tissue dead spaces were left. Surgical wounds of both Group A and Group B patients were closed using Ethilon sutures followed by occlusive dressing using Betadine and gauze in both groups.

\section{Post-operative}

- $\quad$ Group A cases - Dressing was opened after 24 hours and then only open wound treatment by cleaning with Betadine 12 hourly was done till stitch removal.

- $\quad$ Group B cases - Dressing was opened after 48 hours and then again after every 2 days occlusive dressing was done until stitch removal.

- Injection Ceftriaxone $1 \mathrm{gm}$ Intravenous was given to all cases.

Any patient showing signs of SSI - pain at stitch site, redness, fever, swelling, bleeding or discharge from stitch site - were subjected to swab from stitch site for culture sensitivity and antibiotic treatment according to the results of culture and sensitivity.

The stitches of all healthy wounds were removed on 7th post-operative day. Patients were followed up every week for 4 weeks and then as per required according to the surgical procedure/clinical condition.

\section{Results and Discussions}

Out of all 860 cases enrolled in the study, 76 patients showed SSIs. Of this, 32 patients belonged to Group A and 44 patients belonged to group B.

\begin{tabular}{|l|l|l|l|}
\hline & Total cases & SSI cases & Percentage \\
\hline GROUP A & 430 & 32 & $7.44 \%$ \\
\hline GROUP B & 430 & 44 & $10.2 \%$ \\
\hline
\end{tabular}

A Chi-Square Test was performed to understand if Occlusive dressings have an added advantage over Open Wound Treatment in prevention of SSIs. 
Null Hypothesis a0: Occlusive dressing have an added advantage over open wound treatment in the prevention of SSIs.

Alternate Hypothesis $\alpha 1$ : Occlusive dressing have No added advantage over open wound treatment in the prevention of SSIs.

The chi-square statistic is 2.0784 . The p-value is 0.149396 . This result is not significant at $\mathrm{p}<$ 0.05. Hence, we reject the Null Hypothesis.

\section{Conclusions and Recommendations}

From the present study, we conclude that Occlusive Dressings have no added advantage over Open Wound Treatment in the prevention of SSIs and hence Open wound treatment can be taken as an alternative for occlusive dressings.

\section{Acknowledgements}

I sincerely thank the Dean and Ethical committee of Mayo Institute of Medical Sciences for permitting me to conduct this study. I would like to thank all the patients who gave their consent for sharing their medical history and allowing us to use the results from their respective operative procedures for the purpose of this study. I would also like to acknowledge the support of Mr. Saksham Kumar who is an MBA from Asian Institute of Management, The Philippines. He contributed with the validation of statistical methods and statistical results of this study.

\section{References}

[1] A review of prevention of surgical site infections in Indian hospitals based on global guidelines for the prevention of surgical site infection, 2016. J Patient Saf Infect Control 2018;6:1-12 - Arora A, Bharadwaj P, Chaturvedi H, Chowbey P, Gupta S, Leaper D, Mani G K, S Marya S K, Premnath R, Quadros K, Srivastava A, Tendolkar A.

[2] A Prospective Randomized Trial of Open Wound Treatment vs Occlusive Dressings in Elective Surgical Cases with Respect to Surgical Site Infections - Anmol Grover, Amandeep Singh, D. S. Sidhu

[3] Gurrappa, Occlusive vs Gauze Dressings for Local Wound Care in Surgical Patients - Dirk T. Ubbink, MD, PhD; Hester Vermeulen, RN, PhD; Astrid Goossens, RN, PhD; et al

[4] Wound Dressings and Comparative Effectiveness Data - Aditya Sood, Mark S. Granick and Nancy L. Tomaselli

\footnotetext{
*Corresponding author.

E-mail address: arvindpms@ yahoo.co.in
} 